\title{
Improved termination analysis of CHR using self-sustainability analysis
}

\author{
Paolo Pilozzi ${ }^{\star}$ and Danny De Schreye \\ Dept. of Computer Science, K.U.Leuven, Belgium \\ paolo.pilozzi@cs.kuleuven.be danny.deschreye@cs.kuleuven.be
}

\begin{abstract}
In the past few years, several successful approaches to termination analysis of Constraint Handling Rules (CHR) have been proposed. In parallel to these developments, for termination analysis of Logic Programs (LP), recent work has shown that a stronger focus on the analysis of the cycles in the strongly connected components (SCC) of the program is very beneficial, both for precision and efficiency of the analysis.

In this paper we investigate the benefit of using the cycles of the SCCs of CHR programs for termination analysis. It is a non-trivial task to define the notion of a cycle for a CHR program. We introduce the notion of a self-sustaining set of CHR rules and show that it provides a natural counterpart for the notion of a cycle in LP. We prove that non-selfsustainability of an SCC in a CHR program entails termination for all queries to that SCC. Then, we provide an efficient way to prove that an $\mathrm{SCC}$ of a CHR program is non-self-sustainable, providing an additional, new way of proving termination of (part of) the program.

We integrate these ideas into the CHR termination analyser CHRisTA and demonstrate by means of experiments that this extension significantly improves both the efficiency and the performance of the analyser.
\end{abstract}

\section{Introduction}

Termination analysis techniques for Logic Programs often make use of dependency analysis. In some approaches (e.g. [5]), this is done in order to detect (mutually) recursive predicates. For non-recursive predicates, no termination proof is needed, so that termination conditions are only expressed and verified for the recursive ones. In other approaches (e.g. [11], [17]) the termination conditions are explicitly expressed in terms of cycles in the strongly connected components (SCCs) of the dependency graph. It was shown in [17] that such an approach is both efficient and precise. On the benchmark of the termination analysis competition (see [22]) it outperformed competing systems.

Recently, in [23] and [12], we adapted termination analysis techniques from Logic Programming (LP) to Constraint Handling Rules (CHR) $[1,8]$ and developed the CHR termination analyser CHRisTA [13]. These techniques and system are based on recursion, rather than on cycles in SCCs.

The main motivation that led to the current work is that we wanted to adapt the approach based on SCCs to CHR, with the hope of improving efficiency and precision. It turned out that there were considerable complications to achieve this, caused by the fact that for CHR a useful notion of "cycle" is hard to define.

\footnotetext{
* Supported by I.W.T. and F.W.O. Flanders - Belgium
} 
Consider for example the CHR program, $P$, consisting of the CHR rule,

$$
R @ a, b \Leftrightarrow a .
$$

For each application of $R$, two constraints, $a$ and $b$, are removed, while a new constraint, $a$, is added. Since $R$ adds constraints that are required to fire $R$, the application of $R$ depends on itself. An SCC analysis on the dependency graph of $P$ would therefore consider $R$ to be cyclic, while in fact, for every application of $R$, a constraint $b$ is removed and never added again. Therefore, at some point in a computation of $P$, the $b$ constraints will be depleted and thus $P$ will terminate.

A more accurate analysis of cycles in CHR could therefore still rely on an SCC analysis, but could also verify for each SCC whether there exists a dependency for each head of the rules of a component, provided for by the component itself.

Unfortunately, such an approach is still not very accurate. Consider for example the CHR program, $P$, consisting of the rule,

$$
R @ a, a \Leftrightarrow a .
$$

For $R$, which is part of an SCC, both heads are provided for from within the component itself. However, for every application of $R$, two constraints $a$ are removed, while only one constraint $a$ is added. Repeated application of $R$ will therefore result in depletion of these $a$ constraints, and thus $P$ will terminate.

Therefore, in order for an SCC in CHR to be cyclic, we must guarantee that the component is self-sustainable. That is, when the rules of an SCC are applied, they must provide the constraints to fire the same rules again.

In fact, in definite LP, an SCC of the dependency graph of a definite LP program is self-sustainable by default. The reason for this is that definite LP is a single-headed language. Therefore, each cycle in the dependency graph of a definite LP program - as described by the SCCs of the program - corresponds to rule applications that have the potential to be repeated.

In CHR, to characterise the notion of a self-sustainable SCC, we need to identify the multisets of rule applications of an SCC that can be repeated. Consider for example the CHR program, $P$ :

$$
R_{1} @ a, a \Leftrightarrow b, b . \quad R_{2} @ b \Leftrightarrow a .
$$

As can be verified, it is non-terminating. That is, when $R_{1}$ is applied once, it adds two $b$ constraints, allowing $R_{2}$ to be applied twice. By applying $R_{2}$ twice, the input required for application of $R_{1}$ is provided for, thus resulting in a loop.

In this paper, we introduce the notion of self-sustainability for a set of CHR rules. It provides a rather precise approximation of cyclic behaviour in CHR. We characterise self-sustainability for a set of rules by means of a system of linear inequalities. Each solution to this system identifies a multiset based on the given rules, such that, if it is traversed - all rules in the multiset are applied onceit provides all required constraints to potentially traverse the multiset again.

Unfortunately, we cannot use this concept directly as a basis for termination analysis, similar to [11] for LP. The reason is that for any cyclic SCC in a CHR program, the system has infinitely many solutions. In [15], we describe a rather inefficient approach to cope with this problem, resulting in little gain in precision. 
Alternatively, we can use the concept of self-sustainability in a "negative" way. If we can show that an SCC in a CHR program is not self-sustainable - the system has no solution - then this proves that the rules involved in that SCC cannot be part of an infinite computation. Therefore, we can ignore these rules in the termination analysis. By integrating this idea in the CHRisTA analyser, it turns out that precision and efficiency of the analysis is improved.

Our approach will be restricted to the abstract CHR semantics. It is however widely applicable as most other CHR semantics [18] are instances of the abstract CHR semantics. That is, if an SCC of a CHR program is not self-sustainable for the abstract semantics, it cannot be self-sustainable for any more refined semantics. Note that, under the abstract semantics, the two different kinds of rules - simpagation and propagation rules - of a CHR program behave as simplification rules. Hence, we discuss our approach in terms of simplification only.

Finally, note that a CHR program may contain propagation rules and that these rules do not remove the constraints on which they fire. Under the abstract CHR semantics, propagation rules are infinitely applicable on the same combination of constraints. Represented as a simplification rule, they explicitly replace the constraints on which they fire and thus are considered self-sustainable by default. Our approach can therefore only prove non-self-sustainability of an SCC without propagation. In Section 6 , we provide intuitions regarding selfsustainability under the theoretical CHR semantics [8]. This refinement of the abstract CHR semantics additionally considers a fire-once policy on propagation rules to overcome the infinite applicability of propagation rules.

Overview of the paper. In Section 2, we discuss the syntax and semantics of abstract CHR. Section 3 defines CHR dependency graphs for an SCC analysis and CHR nets - a more accurate description of the dependencies of a CHR program - for a self-sustainability analysis. In Section 4, we formalise the notion of self-sustainability of an SCC (Section 4.1). Then, we present a test for verifying that an SCC is not self-sustainable (Section 4.2). In Section 5, we evaluate our approach and discuss CHR programs that we can only prove terminating using the test for non-self-sustainability. Finally, in Section 6, we conclude the paper.

\section{Preliminaries}

We assume familiarity with LP and its main results $[2,9]$. By $L$, we denote the first order language underlying a CHR program $P$. By $\operatorname{Term}_{P}$ and Atom $_{P}$, we denote, respectively, the sets of terms and atoms constructible from $L$.

\subsection{The syntax of abstract CHR}

The rules of a CHR program act on first-order atoms, called constraints.

Definition 1 (constraint). Constraints, $c\left(t_{1}, \ldots, t_{n}\right)(n \geq 0)$, are first-order predicates applied to terms, $t_{i}(1 \leq i \leq n)$. We distinguish between two kinds of constraints: CHR constraints are user-defined and are solved by the CHR rules of a CHR program; built-in constraints are pre-defined and are solved by $a$ constraint theory (CT) defined in the host language. 
Constraints are kept in a constraint store, of which the behaviour corresponds to a multiset (or bag) of constraints. Therefore, to formalise the syntax and semantics of CHR, we first recall multiset theory $[4,6]$.

A multiset is a tuple, $M_{S}=\left\langle S, m_{S}\right\rangle$, where $S$ is a set, called the underlying set, and $m_{S}$ a multiplicity function, mapping the elements, $e$, of $S$ to natural numbers, $m_{S}(e) \in \mathbb{N}_{0}$, representing the number of occurrences of $e$ in $M_{S}$. Like any function, $m_{S}$ may be represented as a set, $\left\{\left(s, m_{S}(s)\right): s \in S\right\}$. An example of a multiset is $\langle\{a, b\},\{(a, 2),(b, 1)\}\rangle$. We introduce the alternative notation to represent this multiset as $\llbracket a, a, b \rrbracket$.

If a universe $U$ in which the elements of $S$ must live is specified, the definition of a multiset can be simplified to a multiset indicator function. Let $U$ be a universe for a multiset $M_{S}=\left\langle S, m_{S}\right\rangle$. Then, we define the multiset indicator function, $\mu_{S}: U \rightarrow \mathbb{N}$, of $M_{S}$ w.r.t. $U$ as: $\mu_{S}(u)=m_{S}(u)$ if $u \in S$ and $\mu_{S}(u)=0$ if $u \notin S$. Since it is often more favourable to discuss multisets in terms of some universe, we can —as an abuse of notation- denote a multiset $M_{S}=\left\langle S, m_{S}\right\rangle$ also by $\left\langle U, \mu_{S}\right\rangle$, or $\mu_{S}$ if its universe is clear from context.

Furthermore, we will need the notion of a multisubset in the constraint store since the rules of a CHR program will be applied on multisubsets of constraints. Let $U$ be a universe for the multisets $M_{S_{1}}$ and $M_{S_{2}}$. Then, multiset $M_{S_{1}}$ is a multisubset of multiset $M_{S_{2}}$, denoted $M_{S_{1}} \sqsubseteq M_{S_{2}}$, iff the multiset indicator functions, $\mu_{S_{1}}$ and $\mu_{S_{2}}$, w.r.t. $U$, of $M_{S_{1}}$ and $M_{S_{2}}$ respectively, are such that $\mu_{S_{1}}(u) \leq \mu_{S_{2}}(u)$, for all $u$ in $U$. Associated to $\sqsubseteq$, we define strict multisubsets: $M_{S_{1}} \sqsubset M_{S_{2}} \leftrightarrow M_{S_{1}} \sqsubseteq M_{S_{2}} \wedge M_{S_{2}} \nsubseteq M_{S_{1}}$.

We are now ready to recall the syntax of simplification rules.

Definition 2 (abstract CHR program). An abstract CHR program is a finite set of simplification rules, $R_{i}$.

$A$ simplification rule,

$$
R_{i} @ H_{1}^{i}, \ldots, H_{n_{i}}^{i} \Leftrightarrow G_{1}^{i}, \ldots, G_{k_{i}}^{i} \mid B_{1}^{i}, \ldots, B_{l_{i}}^{i}, C_{1}^{i}, \ldots, C_{m_{i}}^{i} .
$$

is applicable on a multiset of CHR constraints, matching with the head, $\llbracket H_{1}^{i}, \ldots, H_{n_{i}}^{i} \rrbracket$, of the rule, such that the guard, $\llbracket G_{1}^{i}, \ldots, G_{k_{i}}^{i} \rrbracket$, is satisfiable. Upon application, the multiset of constraints matching with the head is replaced by an appropriate instance of the multiset of built-in and CHR constraints, $\llbracket B_{1}^{i}, \ldots, B_{l_{i}}^{i}, C_{1}^{i}, \ldots, C_{m_{i}}^{i} \rrbracket$, from the body of the rule.

Note that the guard, $\llbracket G_{1}^{i}, \ldots, G_{k_{i}}^{i} \rrbracket$, of a CHR rule may only consist of built-in constraints. Also note that naming a rule by "rulename@” is optional.

The next example program is an introductory example to CHR(Prolog).

Example 1 (Primes). The program below implements the Sieve of Eratosthenes for finding the prime numbers up to a given number. User-defined prime/1 constraints are used to hold the values of the positive integers for which we derive whether they are prime. User-defined primes/1 constraints are used to query the program and to generate the prime/1 constraints for prime evaluation. 


$$
\begin{aligned}
& R_{1} @ \operatorname{primes}(2) \Leftrightarrow \operatorname{prime}(2) . \\
& R_{2} @ \operatorname{primes}(N) \Leftrightarrow N>2 \mid N p \text { is } N-1, \operatorname{prime}(N), \operatorname{primes}(N p) . \\
& R_{3} @ \operatorname{prime}(M), \operatorname{prime}(N) \Leftrightarrow \operatorname{div}(M, N) \mid \operatorname{prime}(M) .
\end{aligned}
$$

$R_{2}$ generates the numbers for prime evaluation top-down. It replaces a $\operatorname{primes}(n)$ constraint, matching with the head $\operatorname{primes}(N)$, by the constraints $N p$ is $n-1$, $\operatorname{prime}(n)$ and $\operatorname{primes}(N p)$, if $C T \models n>2 . R_{1}$ removes a primes $(2)$ constraint, replacing it with the final number for prime evaluation, prime $(2) . R_{3}$ implements the sieve. For any two matching constraints, prime $(m)$ and prime $(n)$, such that $m$ divides $n$, we replace both prime/1 constraints by $\operatorname{prime}(m)$.

\subsection{The semantics of abstract CHR}

The abstract CHR semantics is defined as a state transition system.

Definition 3 (abstract CHR state). An abstract CHR state is a multiset, $Q$, of constraints (with universe Atom $_{P}$ ), called a constraint store.

To define the transitions on CHR states, as given by the CHR rules of the program, we introduce the multiset operator join, adding multisets together. Let $M_{A}$ and $M_{B}$ be multisets with universe $U$, and let $u$ be an element of $U$. Then, the multiset indicator function of the join $M_{C}=M_{A} \uplus M_{B}$ is given by the sum, $\mu_{C}(u)=\mu_{A}(u)+\mu_{B}(u)$, of the multiset indicator functions of $M_{A}$ and $M_{B}$.

The CHR transition relation represents the consecutive CHR states for a CHR program $P$ and constraint theory $C T$.

Definition 4 (abstract CHR transition relation). Let $P$ be a CHR program and $C T$ a constraint theory for the built-in constraints. Let $\theta$ represent the substitutions corresponding to the bindings generated when resolving built-in constraints by $C T$, and let $\sigma$ represent substitutions for the variables in the head of the rule as a result of matching. Then, the transition relation, $\rightarrow_{P}$, for $P$, where $Q$ is a CHR state, is defined by:

\section{A solve transition:}

if $Q=\llbracket b \rrbracket \uplus Q^{\prime}$, with $b$ a built-in constraint, and $C T \models b \theta$,

then, $Q \rightarrow_{P} Q^{\prime} \theta$.

\section{Simplification:}

$$
\begin{aligned}
& \text { if }\left(R_{i} @ H_{1}^{i}, \ldots, H_{n_{i}}^{i} \Leftrightarrow G_{1}^{i}, \ldots, G_{k_{i}}^{i} \mid B_{1}^{i}, \ldots, B_{l_{i}}^{i}, C_{1}^{i}, \ldots, C_{m_{i}}^{i} .\right) \in P, \\
& \text { and if } Q=\llbracket h_{1}, \ldots, h_{n_{i}} \rrbracket \uplus Q^{\prime} \text { and } \\
& \quad \exists \sigma \theta: C T \models\left(h_{1}=H_{1}^{i} \sigma\right) \wedge \ldots \wedge\left(h_{n_{i}}=H_{n_{i}}^{i} \sigma\right) \wedge\left(G_{1}^{i}, \ldots, G_{k_{i}}^{i}\right) \sigma \theta, \\
& \quad \text { then, } Q \rightarrow_{P}\left(\llbracket B_{1}^{i}, \ldots, B_{l_{i}}^{i}, C_{1}^{i}, \ldots, C_{m_{i}}^{i} \rrbracket \uplus Q^{\prime}\right) \sigma \theta \text {. }
\end{aligned}
$$

Rules in CHR are non-deterministically applied until exhaustion, thus until no more transitions are possible. Rule application is a committed choice. Built-in constraints are assumed to return an answer in finite time and cannot introduce new CHR constraints. If built-ins cannot be solved by CT, the program fails.

By an initial CHR state or query state for a CHR program $P$, we mean any abstract $\mathrm{CHR}$ state for $P$. By a final $C H R$ state or answer state for $P$, we mean a CHR state $Q$, such that no CHR state $Q^{\prime}$ exists for which $\left(Q, Q^{\prime}\right) \in \rightarrow_{P}$. 
Example 2 (Primes continued). Executing the program from Example 1 on an initial CHR state $Q_{0}=\llbracket$ primes $(7) \rrbracket$, we obtain by application of $R_{2}$ the next state $Q_{1}=\llbracket N p$ is $7-1$, prime $(7)$, primes $(N p) \rrbracket$. Solving the built-in constraint, $N p$ is $7-1$, binds 6 to $N p$, yielding the state, $Q_{2}=\llbracket p r i m e(7)$, primes $(6) \rrbracket$. Proceeding in this way, we obtain the state $Q_{10}=\llbracket$ prime $(3), \operatorname{primes}(2)$, prime $(4)$, prime (5), prime $(6)$, prime $(7) \rrbracket$, on which $R_{2}$ is no longer applicable. Then, by application of $R_{1}$, we obtain $Q_{11}=\llbracket \operatorname{prime}(2), \operatorname{prime}(3), \operatorname{prime}(4), \operatorname{prime}(5)$, prime $(6), \operatorname{prime}(7) \rrbracket$.

Now only $R_{3}$ is applicable, replacing two prime/1 constraints by the constraint dividing the other. For example, we can remove prime(4) since prime(2) divides it. A next state could therefore be $Q_{12}=\llbracket \operatorname{prime}(2)$, prime $(3)$, prime $(5)$, prime(6), prime(7)】. For prime(6) there are two prime numbers that divide it. One possibility is that prime (3) is used, and obtain $Q_{13}=\llbracket \operatorname{prime}(3), \operatorname{prime}(2)$, prime $(5)$, prime $(7) \rrbracket$.

Since on $Q_{13}$ no more rules are applicable, we reach an answer state. This state holds all prime numbers up to 7.

\section{The CHR dependency graph and CHR net}

In CHR, the head constraints of a rule represent the constraints required for rule application. The body CHR constraints represent the constraints newly available after rule application. To represent these sets, we introduce abstract CHR constraints.

Definition 5 (abstract CHR constraint). An abstract CHR constraint is a pair $\mathcal{C}=(C, B)$, where $C$ is a CHR constraint, and $B$ a conjunction of built-in constraints. The denotation of an abstract $C H R$ constraint is given by a mapping $\wp:(C, B) \mapsto\{C \sigma \mid \exists \theta: C T \models B \sigma \theta\}$.

In a CHR program $P$, we distinguish between two types of abstract CHR constraints. An abstract input constraint, $i n_{j}^{i}=\left(H_{j}^{i}, G^{i}\right)$, represents the CHR constraints that can be used to match the head $H_{j}^{i}$ of a CHR rule $R_{i}$, without invalidating the guards $G^{i}=G_{1}^{i} \wedge \cdots \wedge G_{k_{i}}^{i}$ of $R_{i}$. An abstract output constraint, out $_{j}^{i}=\left(C_{j}^{i}, G^{i}\right)$, represents the CHR constraints that become newly available after rule application, and are related to the body CHR constraints $C_{j}^{i}$ of $R_{i}$.

By $\mathcal{I} n_{R_{i}}$ and $\mathcal{O} u t_{R_{i}}$, we represent the sets of abstract input and output constraints of a rule $R_{i}$. By $\mathcal{I} n_{P}$ and $\mathcal{O} u t_{P}$, we represent the abstract input and output constraints of a CHR program $P$.

Example 3 (abstract CHR constraints of Primes). We revisit the Primes program from Example 1, and derive:

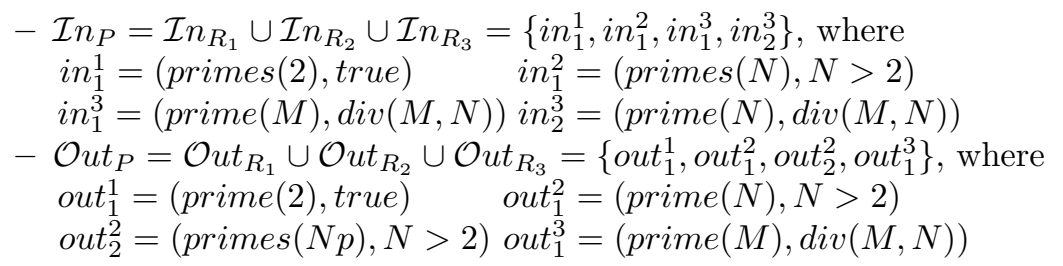


The rules of a CHR program $P$ relate abstract inputs to abstract outputs. We call this relation the rule transition relation of $P$.

Definition 6 (rule transition relation). A rule transition of an abstract CHR program, $P$, is an ordered pair $T_{i}=\left(\mathcal{I} n_{R_{i}}, \mathcal{O} u t_{R_{i}}\right)$, relating the set of abstract input constraints $\mathcal{I} n_{R_{i}}=\left\{i n_{1}^{i}, \ldots, i n_{n_{i}}^{i}\right\}$ of $R_{i} \in P$ to the set of abstract output constraints $\mathcal{O} u t_{R_{i}}=\left\{\right.$ out $_{1}^{i}, \ldots$, out $\left._{m_{i}}^{i}\right\}$ of $R_{i}$. The rule transition relation $\mathcal{T}_{P}=\left\{T_{i} \mid R_{i} \in P\right\}$ of $P$ is the set of rule transitions of $P$.

Example 4 (rule transition relation of Primes). The Primes program $P$ from Example 1 defines three rule transitions:

$-\mathcal{T}=\left\{T_{1}, T_{2}, T_{3}\right\}$, where

$T_{1}=\left(\left\{\right.\right.$ in $\left._{1}^{1}\right\},\left\{\right.$ out $\left.\left._{1}^{1}\right\}\right) \quad T_{2}=\left(\left\{\right.\right.$ in $\left._{1}^{2}\right\},\left\{\right.$ out $_{1}^{2}$, out $\left.\left._{2}^{2}\right\}\right)$

$T_{3}=\left(\left\{i n_{1}^{3}, i n_{2}^{3}\right\},\left\{o u t_{1}^{3}\right\}\right)$

Abstract output constraints relate to abstract input constraints by a match transition relation. This second kind of relation is the result of a dependency analysis between abstract constraints, relating the constraints newly available after rule application to constraints required for rule application.

Definition 7 (match transition relation). A match transition of an abstract CHR program $P$ is an ordered pair $M_{(i, j, k, l)}=\left(\right.$ out $\left._{j}^{i}, i n_{l}^{k}\right)$, relating an output out $_{j}^{i}=\left(C_{j}^{i}, G^{i}\right)$ of $\mathcal{O u t}_{P}$ to an input in $n_{l}^{k}=\left(C_{l}^{k}, G^{k}\right)$ of $\mathcal{I}_{P}$ such that $\exists \theta$ : $C T \models\left(C_{j}^{i}=C_{l}^{k} \wedge G^{i} \wedge G^{k}\right) \theta$. The match transition relation $\mathcal{M}_{P}$ is the set of all match transitions $M_{(i, j, k, l)}$ in $P$.

Note that a match transition exists for an abstract output and input if the intersection of their denotation is non-empty, i.e. $\wp\left(o u t_{j}^{i}\right) \cap \wp\left(i n_{l}^{k}\right) \neq \emptyset$.

Example 5 (match transition relation of Primes). The Primes program, $P$, from Example 1 defines the match transition relation,

$$
\begin{aligned}
& \mathcal{M}_{P}=\left\{M_{(1,1,3,1)}, M_{(1,1,3,2)}, M_{(2,1,3,1)}, M_{(2,1,3,2)}\right. \\
& \left.M_{(2,2,1,1)}, M_{(2,2,2,1)}, M_{(3,1,3,1)}, M_{(3,1,3,2)}\right\} .
\end{aligned}
$$

As in LP, the dependency graph of a CHR program is a directed graph where the nodes represent rules, and the directed arcs dependencies between these rules.

Definition 8 (CHR dependency graph). A CHR dependency graph, $\mathcal{D}_{P}$, of an abstract CHR program $P$ is an ordered tuple $\langle T, D\rangle$ of nodes $T$, one for each transition in the rule transition relation, $\mathcal{T}_{P}$, of $P$, and directed arcs $D$, one for each ordered pair of transitions between which a match transition exists in the match transition relation, $\mathcal{M}_{P}$, of $P$.

For an SCC analysis, a CHR dependency graph is sufficient, however, for self-sustainability we rely in the next sections on a CHR net instead.

Definition 9 (CHR net). $A$ CHR net, $\mathcal{N}_{P}$, of an abstract $C H R$ program, $P$, is a quadruple $\left\langle\mathcal{I} n_{P}, \mathcal{O} u t_{P}, \mathcal{T}_{P}, \mathcal{M}_{P}\right\rangle$. Here, respectively, $\mathcal{I} n_{P}$ and $\mathcal{O} u t_{P}$ are the abstract input and output constraints of $P$, and $\mathcal{T}_{P}$ and $\mathcal{M}_{P}$ the rule and match transition relations of $P$. 
Note that a CHR net corresponds to a bipartite hypergraph. We illustrate both notions in Figure 1 for the Primes program of Example 1.

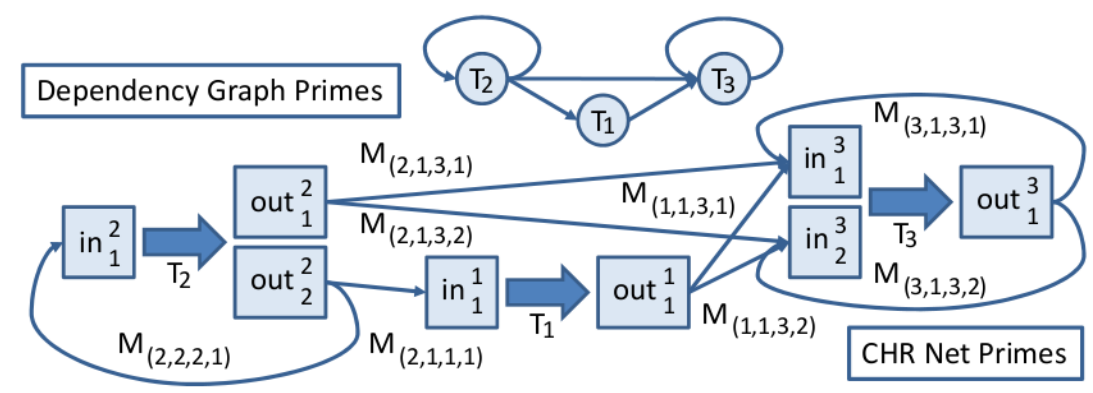

Fig. 1. Dependency graph and CHR net for Primes

\section{Self-sustainable SCCs of a CHR program}

To derive the SCCs of a CHR program, we perform an SCC analysis on the dependency graph of the CHR program. This is similar to such an analysis in LP and can be done efficiently using Tarjan's algorithm [21].

Example 6 (SCCs of Primes). Based on $\mathcal{D}_{P}=\left(\left\{T_{1}, T_{2}, T_{3}\right\},\left\{\left(T_{1}, T_{3}\right),\left(T_{2}, T_{1}\right)\right.\right.$, $\left.\left.\left(T_{2}, T_{2}\right),\left(T_{2}, T_{3}\right),\left(T_{3}, T_{3}\right)\right\}\right)$, the dependency graph of Primes of Figure 1 , we derive two SCCs, $\left\{T_{2}\right\}$ and $\left\{T_{3}\right\}$.

\subsection{Self-sustainable SCCs}

Consider again the non-terminating example program from the Introduction:

$$
R_{1} @ a, a \Leftrightarrow b, b . \quad R_{2} @ b \Leftrightarrow a .
$$

Its dependency graph and CHR net are shown in Figure 2. As can be verified, in Figure $2, i n_{1}^{1}=i n_{2}^{1}=o u t_{1}^{2}=(a$, true $)$ and out $t_{1}^{1}=o u t_{2}^{1}=i n_{1}^{2}=(b$, true $)$. For the example, there is only one SCC: $\left\{T_{1}, T_{2}\right\}$.
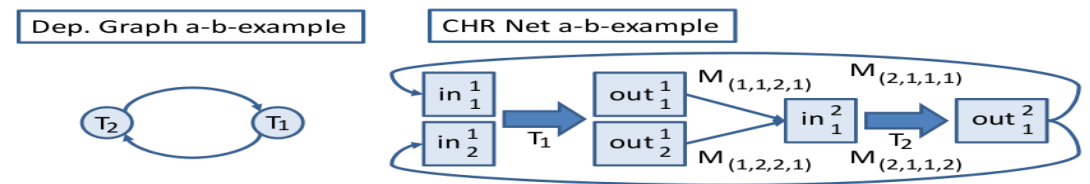

Fig. 2. Dependency graph and CHR net for the $a$-b-example

What we want to characterise - in the notion of self-sustainability for such an SCC - is whether we can duplicate some of the transitions of this SCC, such that for the resulting multiset of transitions and its extended CHR net, it is so that a multisubset of all $M_{(i, j, k, l)}$ match transitions exists, that maps a multisubset of all the out ${ }_{q}^{p}$ nodes onto all of the $i n_{s}^{r}$ nodes. Returning to our example, consider 
the multiset $\llbracket T_{1}, T_{2}, T_{2} \rrbracket$ based on $\left\{T_{1}, T_{2}\right\}$. In Figure 3 , we expand the CHR net of Figure 2 to represent all match transitions for this multiset.

It is clear that from the multiset of 8 match transitions in Figure 3, we can select 4 transitions, for example $\llbracket M_{(1,1,2,1)}, M_{(1,2,2,1)}, M_{(2,1,1,1)}, M_{(2,1,1,2)} \rrbracket$, such that these transitions define a function from a multisubset of all out $t_{q}^{p}$ nodes onto all $i n_{s}^{r}$ nodes. This function is represented in Figure 3 in the thick arrows.

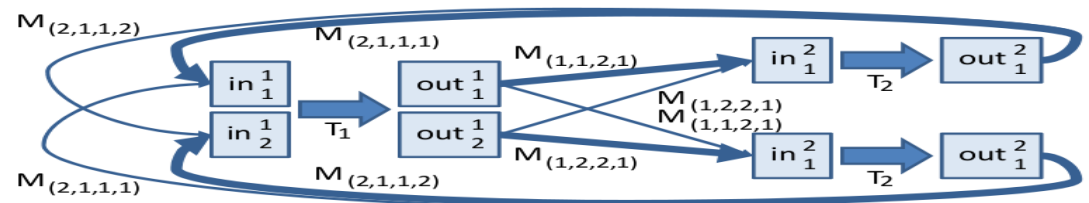

Fig. 3. Expanded CHR net for $\llbracket T_{1}, T_{2}, T_{2} \rrbracket$

Note that in this example, the multisubsets of outp nodes on which the function is defined is equal to the entire multiset, $\llbracket$ out $t_{1}^{1}$, out $t_{2}^{1}$, out $_{1}^{2}$, out $_{1}^{2} \rrbracket$, of out $t_{q}^{p}$ nodes. In general, this is not necessary: out $t_{q}^{p}$ nodes are allowed to be "unused" by $i n_{s}^{r}$ nodes. It suffices that a CHR constraint is produced for each $i n_{s}^{r}$ node.

We generalise the construction in the example above in the following definition. In this definition, we start off from a set of CHR rules, $C$, and its CHR net, $\mathcal{N}_{C}=\left\langle\mathcal{I}_{C}, \mathcal{O u t}_{C}, \mathcal{T}_{C}, \mathcal{M}_{C}\right\rangle$. In the definition we will consider a multiset with universe the rule transitions $\mathcal{T}_{C}$, and will denote it by $\mu_{\mathcal{T}}$. Note that, given such a multiset $\mu_{\mathcal{T}}$, there are associated multisets $\mu_{\mathcal{I} n}$ and $\mu_{\mathcal{O} u t}$, with universes $\mathcal{I} n_{C}$ and $\mathcal{O} u t_{C}$, respectively. If a transition $T_{i} \in \mathcal{T}_{C}$ occurs $n_{i}$ times in $\mu_{\mathcal{T}}$, then all its $i n_{j}^{i}$ and out $_{l}^{i}$ abstract constraints occur $n_{i}$ times in $\mu_{\mathcal{I} n}$ and $\mu_{\mathcal{O} u t}$, respectively.

Given $\mu_{\mathcal{T}}$, there is also an associated multiset $\mu_{\mathcal{M}}$, with universe $\mathcal{M}_{C}$. If transitions $T_{i}$ and $T_{k}$ occur respectively $n_{i}$ and $n_{k}$ times in $\mu_{\mathcal{T}}$, then all $M(i, j, k, l) \in \mathcal{M}_{C}$ occur $n_{i} \times n_{k}$ times in $\mu_{\mathcal{M}}$.

Definition 10 (self-sustainability of a set of CHR rules). Let $C$ be a set of simplification rules and $\mathcal{N}_{C}=\left\langle\mathcal{I} n_{C}, \mathcal{O} u t_{C}, \mathcal{T}_{C}, \mathcal{M}_{C}\right\rangle$ the CHR net of $C$. The set $C$ is self-sustainable iff there exist

- a non-empty multiset $\mu_{\mathcal{T}}$, with the associated multisets $\mu_{\mathcal{I} n}, \mu_{\mathcal{O} \text { ut }}$ and $\mu_{\mathcal{M}}$,

- a multiset $\mu_{\mathcal{O} u t}^{\prime} \sqsubseteq \mu_{\mathcal{O} u t}$, and

- a multiset $\mu_{\mathcal{M}}^{\prime} \sqsubseteq \mu_{\mathcal{M}}$,

such that $\mu_{\mathcal{M}}^{\prime}$ defines a function from $\mu_{\mathcal{O} u t}^{\prime}$ onto $\mu_{\mathcal{I} n}$.

Before we illustrate this concept on our running example, Primes, we provide an alternative, numeric characterisation.

Proposition 1 (numeric characterisation self-sustainability). Let $C$ be a set of simplification rules and $\mathcal{N}_{C}=\left\langle\mathcal{I}_{C}, \mathcal{O} u t_{C}, \mathcal{T}_{C}, \mathcal{M}_{C}\right\rangle$ its CHR net. The set $C$ is self-sustainable iff there exist multisets $\mu_{\mathcal{T}}$, with associated multisets $\mu_{\mathcal{I} n}$, 
$\mu_{\mathcal{O} u t}, \mu_{\mathcal{M}}, \mu_{\mathcal{O} u t}^{\prime} \sqsubseteq \mu_{\mathcal{O} u t}$ and $\mu_{\mathcal{M}}^{\prime} \sqsubseteq \mu_{\mathcal{M}}$, such that $\sum_{i: T_{i} \in \mathcal{T}_{C}} \mu_{\mathcal{T}}\left(T_{i}\right) \geq 1$ and such that

$$
\begin{aligned}
& \forall \text { out }_{j}^{i} \in \mathcal{O}_{C}: \sum_{k, l: M_{(i, j, k, l)} \in \mathcal{M}_{C}} \mu_{\mathcal{M}}^{\prime}\left(M_{(i, j, k, l)}\right) \leq \mu_{\mathcal{T}}\left(T_{i}\right), \text { and } \\
& \forall i n_{j}^{i} \in \mathcal{I}_{C}: \sum_{k, l: M_{(k, l, i, j)} \in \mathcal{M}_{C}} \mu_{\mathcal{M}}^{\prime}\left(M_{(k, l, i, j)}\right)=\mu_{\mathcal{T}}\left(T_{i}\right) .
\end{aligned}
$$

Proof. The first inequality expresses that $\mu_{\mathcal{T}}$ is non-empty. The second kind of inequalities - one for each out ${ }_{j}^{i}$ in $\mathcal{O}_{4} t_{C}$ - state that for an abstract output $o u t_{j}^{i}$, the number of match transitions $M_{(i, j, k, l)}$ in $\mu_{\mathcal{M}}^{\prime}$ that have $(i, j)$ as their first two arguments does not exceed the number of occurrences of $T_{i}$ in $\mu_{\mathcal{T}}$. This corresponds to stating that $\mu_{\mathcal{M}}^{\prime}$ can be regarded as a function defined on a multisubset $\mu_{\mathcal{O} u t}^{\prime}$ of $\mu_{\mathcal{O} u t}$.

The third kind of inequalities - one for each $i n_{j}^{i}$ in $\mathcal{I} n_{C}$ - state that for an abstract input constraint $i n_{j}^{i}$, the number of match transitions $M_{(k, l, i, j)}$ in $\mu_{\mathcal{M}}^{\prime}$ that have $(i, j)$ as their last two arguments is exactly the number of occurrences of $T_{i}$ in $\mu_{\mathcal{T}}$. This means that the function defined by $\mu_{\mathcal{M}}^{\prime}$ is onto $\mu_{\mathcal{I} n}$.

Example 7 (self-sustainable SCCs for Primes). Consider the SCCs, $C_{1}=\left\{T_{2}\right\}$ and $C_{2}=\left\{T_{3}\right\}$, (see Example 6 ) of the Primes program, $P$, of Example 1. While the first component of the program is self-sustainable, the second is not. That is, at some point in a computation of $C_{2}$, the prime/1 constraints to fire the rule will be depleted. Consider their CHR nets,

$$
\begin{aligned}
& \mathcal{N}_{C_{1}}=\left\langle\left\{\text { in }_{1}^{2}\right\},\left\{\text { out }_{1}^{2}, \text { out }_{2}^{2}\right\},\left\{T_{2}\right\},\left\{M_{(2,2,2,1)}\right\}\right\rangle \text { and } \\
& \mathcal{N}_{C_{2}}=\left\langle\left\{\text { in }_{1}^{3}, \text { in }_{2}^{3}\right\},\left\{\text { out }_{1}^{3}\right\},\left\{T_{3}\right\},\left\{M_{(3,1,3,1)}, M_{(3,1,3,2)}\right\}\right\rangle .
\end{aligned}
$$

Let us denote $\mu_{\mathcal{M}}^{\prime}\left(M_{(i, j, k, l)}\right)$ as $m_{(i, j, k, l)}$ and $\mu_{\mathcal{T}}\left(T_{i}\right)$ as $t_{i}$, where all $m_{(i, j, k, l)}$ and $t_{i}$ are natural numbers. Then, we can characterise self-sustainability of $C_{1}$ and $C_{2}$, respectively, by the systems of linear inequalities,

$$
\begin{array}{ll}
t_{2} \geq 1 & m_{(2,2,2,1)} \leq t_{2} \quad m_{(2,2,2,1)}=t_{2} \quad \text { and } \\
t_{3} \geq 1 & m_{(3,1,3,1)}+m_{(3,1,3,2)} \leq t_{3} \quad m_{(3,1,3,1)}=t_{3} \quad m_{(3,1,3,2)}=t_{3} .
\end{array}
$$

If a solution to these systems exists, the underlying component is self-sustainable. $C_{1}$ is clearly self-sustainable, while $C_{2}$ is not.

Several comments with respect to Definition 10 and Proposition 1 are in order. First, the statement that $\mu_{\mathcal{M}}^{\prime}$ defines a function from $\mu_{\mathcal{O} u t}^{\prime}$ onto $\mu_{\mathcal{I} n}$ is imprecise. In fact, $\mu_{\mathcal{M}}^{\prime}$ defines a set of functions from $\mu_{\mathcal{O} u t}^{\prime}$ onto $\mu_{\mathcal{I} n}$. This is because $\mu_{\mathcal{O} u t}^{\prime}$ and $\mu_{\mathcal{I} n}$ are multisets, that may contain elements more than once.

Therefore, if $i n_{j}^{i}$ or out $t_{l}^{k}$ occur multiple times in $\mu_{\mathcal{I} n}$, respectively $\mu_{\mathcal{O} u t}^{\prime}$, it is unclear which mapping is defined by an element $M_{(k, l, i, j)}$ of $\mu_{\mathcal{M}}^{\prime}$. Looking back at the $a$-b-example of Figure 3, there are four different functions in this graph, all corresponding to the multiset $\mu_{\mathcal{M}}^{\prime}=\llbracket M_{(1,1,2,1)}, M_{(1,2,2,1)}, M_{(2,1,1,1)}, M_{(2,1,1,2)} \rrbracket$. The thick lines in the figure represent one of these, but selecting other arcs, with the same labels, produces the other three. 
Apart from this, for a fixed multiset $\mu_{\mathcal{T}}$, there can be several mappings $\mu_{\mathcal{M}}^{\prime}$ that map a multiset $\mu_{\mathcal{O} u t}^{\prime}$ onto $\mu_{\mathcal{I} n}$. This is because there can be different abstract constraints out ${ }_{l}^{k}$ in $\mu_{\mathcal{O} u t}$ that all have a match transition to some abstract constraint $i n_{j}^{i}$. This may give a number of alternative candidates for $\mu_{\mathcal{M}}^{\prime}$.

Finally, by considering different multisets $\mu_{\mathcal{T}}$ based on $\mathcal{T}_{C}$, we may obtain a very large number of solutions for $\mu_{\mathcal{M}}^{\prime}$ in Definition 10. In the context of the $a$-b-example, consider a multiset $\mu_{\mathcal{T}}=\llbracket T_{1}, T_{1}, T_{2}, T_{2}, T_{2}, T_{2} \rrbracket$. From the fact that there are twice as many $T_{2}$ rules than $T_{1}$ rules, it should be intuitively clear that it again allows to find multisets $\mu_{\mathcal{O} u t}^{\prime}$ and $\mu_{\mathcal{M}}^{\prime}: \mu_{\mathcal{O} u t}^{\prime} \rightarrow \mu_{\mathcal{I} n}$, with the latter being onto. Because of the increased multiplicity of the rule transitions, the number of different functions that $\mu_{\mathcal{M}}^{\prime}$ represents in this context is much higher than for the previous $\mu_{\mathcal{T}}$. Moreover, it turns out that by increasing the multiplicity of the rule transitions, we can construct concrete functions associated to $\mu_{\mathcal{M}}^{\prime}$ that cannot be obtained as the union of multiple concrete functions associated to a solution for a $\mu_{\mathcal{T}}$ with lower multiplicity of rule transitions (see [14]).

This observation implies that we are unable to use the notion of a selfsustainable set of rules as a direct basis for a termination analysis. Such an approach would have to identify a finite set of minimal self-sustainable cycles and then prove that all these are terminating. But since in CHR, there are in general an infinite set of minimal cycles, such an approach is not feasible.

In [15], to tackle the problem of an infinite number of minimal cycles, a new concept of minimality is introduced, based on a finite constructive set of solutions - the Hilbert basis - for the inequalities representing self-sustainability. This approach is however slow, with little gain in precision.

Fortunately, there is another way for using the notion of a self-sustaining set of rules. After determining the SCCs of a CHR program, we can verify which SCCs are not self-sustainable, and disregard such SCCs. This observation is based on the following theorems.

Theorem 1. If a CHR program, $P$, is not self-sustainable, then $P$ terminates for every query.

Theorem 2. Let $P$ be a CHR program and let $C$ be an $S C C$ of $P$. If $C$ is not self-sustainable and if $P \backslash C$ terminates for every query, then $P$ terminates for every query.

\subsection{Non-self-sustainable SCCs of a CHR program}

From here on, we refer to self-sustainable as 'selfs' and use the matrix form: $A \times X \leq B$; to represent systems of linear inequalities.

Example 8 (system in matrix form for Primes). We revisit Example 7 and represent for $C_{1}$ and $C_{2}$, respectively, their systems of inequalities in matrix form:

$$
\left[\begin{array}{cc}
-1 & 1 \\
1 & -1 \\
-1 & 1 \\
-1 & 0
\end{array}\right] \times\left[\begin{array}{c}
t_{2} \\
m_{(2,2,2,1)}
\end{array}\right] \leq\left[\begin{array}{c}
0 \\
0 \\
0 \\
-1
\end{array}\right] \text { and }\left[\begin{array}{ccc}
-1 & 1 & 0 \\
1 & -1 & 0 \\
-1 & 0 & 1 \\
1 & 0 & -1 \\
-1 & 1 & 1 \\
-1 & 0 & 0
\end{array}\right] \times\left[\begin{array}{c}
t_{3} \\
m_{(3,1,3,1)} \\
m_{(3,1,3,2)}
\end{array}\right] \leq\left[\begin{array}{c}
0 \\
0 \\
0 \\
0 \\
0 \\
-1
\end{array}\right]
$$


Note that the equalities of the original system are replaced by two inequalities. E.g. $m_{(2,2,2,1)}=t_{2}$ is replaced by $m_{(2,2,2,1)} \geq t_{2}$ and $m_{(2,2,2,1)} \leq t_{2}$.

In order to prove that a component is not selfs, we need to prove that no positive integer solution exists for the variables of the linear inequalities representing that it is selfs. Thus, we need to prove that such a system is infeasible.

Definition 11 (feasible). A system, $S=A \times X \leq B$, is (in)feasible iff $S$ has (not) a solution in $\mathbb{R}^{+}$.

The following lemma is due to Farkas [7].

Lemma 1 (Farkas' lemma). Let $S=A \times X \leq B$ be a system of linear inequalities. Then, $S$ is feasible iff $\forall P \geq 0: A^{T} \times P \geq 0 \rightarrow B^{T} \times P \geq 0$. Alternatively, $S$ is infeasible iff $\exists P \geq 0: A^{T} \times P \geq 0 \wedge B^{T} \times P<0$.

Note that Farkas' lemma is only applicable to the real case: if a real matrix $P$ exists, such that $P \geq 0 \wedge A^{T} \times P \geq 0 \wedge B^{T} \times P<0$, then, the original system, $S$, is infeasible. Therefore, it has no solution in $\mathbb{N} \subset \mathbb{R}^{+}$and must be non-selfs.

Infeasibility has received much attention in linear programming (see e.g. [3]) and several approaches exist to tackle the problem. It is not in our intention to improve on these approaches. To evaluate our approach, we do however formulate a simple test, where we first represent infeasibility as a constraint problem on symbolic coefficients. That is, we introduce a symbolic matrix $P^{\prime}$, for each infeasibility problem, of the same dimensions as $P$, with symbolic coefficients $p_{i}$, one for each position in the matrix. Then, we derive constraints on the symbolic coefficients, based on $P^{\prime} \geq 0 \wedge A^{T} \times P^{\prime} \geq 0 \wedge B^{T} \times P^{\prime}<0$.

Example 9 (infeasibility for Primes). We revisit Example 8, and formulate infeasibility of the systems of linear inequalities. That is, let $P_{1}$ for $C_{1}$ be a $(4 \times 1)$ matrix of (integer) symbolic coefficients $p_{0}^{1}, p_{1}^{1}, \ldots, p_{3}^{1}$, all greater or equal to 0 . Then, to prove non-selfs for $C_{1}$, we need to satisfy:

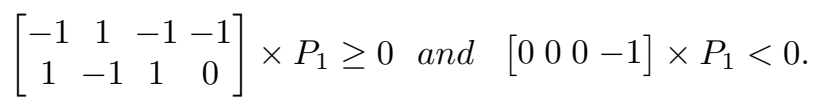

We derive the following problem, where $\forall i \in\{0,1, \ldots, 3\}: p_{i}^{1} \geq 0$ :

$$
-p_{0}^{1}+p_{1}^{1}-p_{2}^{1}-p_{3}^{1} \geq 0 \quad p_{0}^{1}-p_{1}^{1}+p_{2}^{1} \geq 0 \quad-p_{3}^{1}<0
$$

There is no solution to this problem, thus $C_{1}$ is selfs. For $C_{2}$, we have

$$
\left[\begin{array}{cccccc}
-1 & 1 & -1 & 1 & -1 & -1 \\
1 & -1 & 0 & 0 & 1 & 0 \\
0 & 0 & 1 & -1 & 1 & 0
\end{array}\right] \times P_{2} \geq 0 \text { and }\left[\begin{array}{llllll}
0 & 0 & 0 & 0 & 0 & -1
\end{array}\right] \times P_{2}<0 .
$$

We derive the following problem, where $\forall i \in\{0,1, \ldots, 5\}: p_{i}^{2} \geq 0$ :

$$
\begin{array}{ll}
-p_{0}^{2}+p_{1}^{2}-p_{2}^{2}+p_{3}^{2}-p_{4}^{2}-p_{5}^{2} \geq 0 & p_{0}^{2}-p_{1}^{2}+p_{4}^{2} \geq 0 \\
p_{2}^{2}-p_{3}^{2}+p_{4}^{2} \geq 0 & -p_{5}^{2}<0
\end{array}
$$

One solution is: $p_{0}^{2}=0, p_{1}^{2}=1, p_{2}^{2}=0, p_{3}^{2}=1, p_{4}^{2}=1$, and $p_{5}^{2}=1$. Therefore, $\mathrm{C}_{2}$ is non-selfs and thus must be terminating. 
To find a solution to these constraint problems, we can transform them to SAT problems, representing that a component is non-selfs, by using a transformation based on signed integer arithmetic. Thus, in this approach, we can only represent integers and not reals. This is still sufficient to represent the infeasibility problem, however, yields an incomplete approach (see Lemma 1): it is not guaranteed that a solution in the integers can be found if the original system is infeasible. Nevertheless, as it turns out, the approach works well in practice.

\section{Evaluation}

We have implemented the non-selfs test using SWI-Prolog [19] and integrated it with CHRisTA, a termination analyser for CHR(Prolog) [13]. This resulted in a new termination analyser, T-CoP [20] (Termination of CHR on top of Prolog).

CHRisTA uses CHR nets as a representation of CHR programs and implements an SCC analysis based on Tarjan's algorithm. To solve the satisfiability problems that CHRisTA is confronted with, we represent the Diophantine constraints as a sufficient SAT problem based on signed integer arithmetic and solve it with MiniSAT2 [10]. Of course, by doing so, we place limits on the domains of variables and integers of the original problem. That is, we limit on the bit-sizes used for representing the variables and the integers of the problem. We reuse this system to prove infeasibility of the linear inequalities that we obtain for selfs.

Therefore, integrating the non-selfs test into CHRisTA involved the following steps: deriving a system of linear inequalities for selfs from the CHR net of an SCC, formulating infeasibility of that system, and transforming the resulting infeasibility problem to a SAT problem. Afterwards, a proof of termination of the remaining SCCs, that are possibly selfs, is attempted by the termination proof procedure of CHRisTA.

\begin{tabular}{||l||c|c||c|c||c||c||}
\hline CHR program & 3bit & sec & 4bit & sec & CHRisTA (sec) & T-CoP (sec) \\
\hline factorial & - & 0.10 & - & 0.14 & 1.50 & 1.64 \\
mean & + & 0.35 & + & 0.58 & 1.26 & 0.58 \\
mergesortscc1 & - & 0.12 & - & 0.20 & 1.44 & 1.64 \\
mergesortscc2 & + & 0.19 & + & 0.34 & 1.51 & 0.34 \\
newcase1 & + & 0.41 & + & 0.74 & timeout & 0.74 \\
newcase2 & - & 1.09 & + & 2.03 & timeout & 2.03 \\
primesscc1 & - & 0.11 & - & 0.19 & 1.67 & 1.86 \\
primesscc2 & + & 0.18 & + & 0.33 & 1.11 & 0.33 \\
\hline
\end{tabular}

Table 1. Results of non-selfs test on terminating SCCs.

Table 1 contains a representative set of the results that we obtained. T-CoP, as well as the full benchmark, is available online for reference [20]. Note that the considered programs of Table 1 contain only a single SCC of a size and complexity similar to the SCCs expected in real-sized CHR programs.

In Table 1, there are SCCs that are non-selfs, which are proven terminating (+) using at most a 4 bit representation for the variables and integers of the SAT problems. The SCCs, only proven terminating by CHRisTA, are selfs.

Among these programs, there are two programs on which the proof procedure of CHRisTA fails (see [12]). An example, representative for such programs, is 
$\left\{\left(R_{1} @ a, a \Leftrightarrow b.\right),\left(R_{2} @ b \Leftrightarrow a\right.\right.$. $\left.)\right\}$. To prove termination, for $R_{1}$, the size of $a$ must be greater or equal to $b$. For $R_{2}, a$ has to be strictly smaller than $b$.

Our new approach can handle such programs, and integrated with CHRisTA, improves the precision of the termination analysis as shown in Table 1 under "TCoP". From these results we may also conclude that the non-selfs test improves the efficiency of the analysis: If an SCC cannot be proven non-selfs, the overhead is acceptable; if it can be proven non-selfs, the gain in speed is relatively high.

Finally, note that the transformational approach of [16], combined with the technique of [17], can be used to handle programs such as newcase 1 and 2 . Unfortunately, in the presence of propagation, the transformational approach of [16] cannot be applied.

\section{Conclusion and future work}

In this paper, we developed an approach to detect non-self-sustainability of the SCCs of a CHR program by means of a satisfiability problem. Integrating the approach with CHRisTA [13], resulted in a more efficient analyser, T-CoP. For one, the approach improves the precision of the termination analysis, being able to prove termination of a new class of CHR programs automatically. Furthermore, we improve the efficiency of the termination analyser CHRisTA (cfr. T-CoP).

Future work will consider propagation rules. For self-sustainability of propagation, we will need to assume presence of a fire-once policy. Intuitively, under such a policy, if no new combinations of constraints are ever introduced as a consequence of applying a propagation rule, such that the propagation rule can be applied again on the newly introduced combinations of constraints, then the propagation rule is non-selfs. Informally, this corresponds to verifying whether there does not exist a cycle in the CHR net of a CHR program across an abstract input constraint of the propagation rule and across some abstract output constraint added by a rule of the program. If so, the propagation rule can be ignored. Therefore, if we obtain by this approach an SCC that ultimately does not contain propagation, then, we can verify whether the SCC is non-selfs by the approach of this paper. Otherwise, the remaining SCC is still selfs by default.

\section{References}

1. Slim Abdennadher. Operational semantics and confluence of constraint propagation rules. In Principles and Practice of Constraint Programming - CP97, Third International Conference, Linz, Austria, October 29 - November 1, 1997, Proceedings, pages 252-266, 1997.

2. Krzysztof R. Apt. Logic programming. In Handbook of Theoretical Computer Science, Volume B: Formal Models and Sematics (B), pages 493-574. 1990.

3. J. E. Beasley, editor. Advances in linear and integer programming. Oxford University Press, Inc., 1996.

4. Wayne D. Blizard. Multiset theory. Notre Dame Journal of Formal Logic, 30(1):3666, 1989.

5. Stefaan Decorte, Danny De Schreye, and Henk Vandecasteele. Constraint-based termination analysis of logic programs. ACM Trans. Program. Lang. Syst., 21(6):1137-1195, 1999. 
6. Nachum Dershowitz and Zohar Manna. Proving termination with multiset orderings. Commun. ACM, 22(8):465-476, 1979.

7. Julius G. Farkas. Über die theorie der einfachen ungleichungen. Journal für die Reine und Angewandte Mathematik, 124:1-27, 1902.

8. Thom W. Frühwirth. Theory and practice of constraint handling rules. J. Log. Program., 37(1-3):95-138, 1998.

9. John W. Lloyd. Foundations of Logic Programming, 2nd Edition. Springer, 1987.

10. MiniSAT, 2010. http://minisat.se/.

11. Manh Thang Nguyen, Jürgen Giesl, Peter Schneider-Kamp, and Danny De Schreye. Termination analysis of logic programs based on dependency graphs. In Logic-Based Program Synthesis and Transformation, 17th International Symposium, LOPSTR 2007, Kongens Lyngby, Denmark, August 23-24, 2007, Revised Selected Papers, pages 8-22, 2007.

12. Paolo Pilozzi and Danny De Schreye. Termination analysis of CHR revisited. In Logic Programming, 24th International Conference, ICLP 2008, Udine, Italy, December 9-13 2008, Proceedings, pages 501-515, 2008.

13. Paolo Pilozzi and Danny De Schreye. Automating termination proofs for CHR. In Logic Programming, 25th International Conference, ICLP 2009, Pasadena, CA, USA, July 14-17, 2009. Proceedings, pages 504-508, 2009.

14. Paolo Pilozzi and Danny De Schreye. Scaling termination proofs by a characterization of cycles in CHR. Technical Report CW 541, K.U.Leuven - Dept. of C.S., Leuven, Belgium, 2009.

15. Paolo Pilozzi and Danny De Schreye. Scaling termination proofs by a characterisation of cycles in CHR. In Termination Analysis, 11th International Workshop on Termination, WST 2010, Edinburgh, United Kingdom, July 14-15, 2010. Proceedings, 2010.

16. Paolo Pilozzi, Tom Schrijvers, and Danny De Schreye. Proving termination of CHR in Prolog: A transformational approach. In Termination Analysis, 9th International Workshop on Termination, WST 2007, Paris, France, June, 200\%. Proceedings, 2007.

17. Peter Schneider-Kamp, Jürgen Giesl, and Manh Thang Nguyen. The dependency triple framework for termination of logic programs. In Logic-Based Program Synthesis and Transformation, 19th International Symposium, LOPSTR 2009, Coimbra, Portugal, September 2009, Revised Selected Papers, pages 37-51, 2009.

18. Jon Sneyers, Peter Van Weert, Tom Schrijvers, and Leslie De Koninck. As time goes by: Constraint Handling Rules - A survey of CHR research between 1998 and 2007. Theory and Practice of Logic Programming, 10(1):1-47, 2010.

19. SWI-Prolog, 2010. http://www.swi-prolog.org.

20. T-CoP, 2010. http://people.cs.kuleuven.be/ paolo.pilozzi?pg=tcop.

21. Robert Endre Tarjan. Depth-first search and linear graph algorithms. SIAM J. Comput., 1(2):146-160, 1972.

22. Termination Competition, 2010. http://termination-portal.org/.

23. Dean Voets, Danny De Schreye, and Paolo Pilozzi. A new approach to termination analysis of constraint handling rules. In Logic Programming, 18th International Symposium on Logic-Based Program Synthesis and Transformation, LOPSTR 2008, Valencia, Spain, July 17-18 2008, Pre-proceedings, pages 28-42, 2008. 